\title{
SYSTEMS OF ALGEBRAIC MIXED DIFFERENCE EQUATIONS*
}

\author{
BY \\ FRITZ HERZOG
}

In his algebraic theory of differential equations, J. F. Ritt $\dagger$ has developed a decomposition theory for systems of algebraic differential equations by introducing the idea of irreducible systems and proving that every system is equivalent to one and essentially only one finite set of irreducible systems. The analogous theorem for algebraic difference equations was given by Ritt and Doob. $\neq$ The purpose of the present paper is to derive a decomposition theorem for algebraic mixed difference equations; i.e., equations which contain algebraically one or more unknown functions $y(x)$, their "transforms" $y(x), y(x+1), y(x+2), \cdots$, and the derivatives of those transforms.

\section{Fields}

Let $\mathfrak{A}$ be an open region in the plane of the complex variable $x$ which has the property that it contains the point $x+1$ whenever $x$ lies in $\mathfrak{A}$. Let $\mathfrak{F}$ be a set of functions, meromorphic in $\mathfrak{A}$. If the following four conditions are satisfied $\mathcal{F}$ will be called a field:

(a) $F$ contains at least one function which is not identically zero.

(b) If $f(x)$ and $g(x)$ are in $\mathcal{F}$, then $f \pm g, f \cdot g$ and $f / g(g \neq 0)$ are in $\mathcal{F}$.

(c) If $f(x)$ is in $\mathcal{F}$ then also $f(x+1)$ is in $\mathfrak{F}$.

(d) If $f(x)$ is in $\mathcal{F}$ then also the derivative of $f(x)$ is in $F$.

\section{FORMS}

We introduce a finite number of letters $y_{1}, y_{2}, \cdots, y_{n}$, which will represent unknown functions $y_{1}(x), y_{2}(x), \cdots, y_{n}(x)$ and will be called unknowns. With every $y_{i}$ we associate the symbols

$$
\begin{array}{rr}
y_{i j}=y_{i}(x+j) & \left(y_{i 0}=y_{i}\right), \\
y_{i j k}=\frac{d^{k} y_{i j}}{d x^{k}}=\frac{d^{k} y_{i}(x+j)}{d x^{k}} & \left(y_{i j 0}=y_{i j}, y_{i 00}=y_{i 0}=y_{i}\right)
\end{array}
$$

* Presented to the Society, October 27, 1934; received by the editors August 9, 1934.

$\dagger$ Ritt, Differential Equations from the Algebraic Standpoint, Colloquium Publications of the American Mathematical Society, vol. 14, 1932.

$\ddagger$ Ritt and Doob, Systems of algebraic difference equations, American Journal of Mathematics, vol. 55 (1933), pp. 505-514. 
where $j$ and $k$ are non-negative integers. $y_{i j}=y_{i}(x+j)$ will be called the $j t h$ transform of the unknown $y_{i}$.

A polynomial in the $y_{i j k}$ the coefficients of which are functions, meromorphic in an open region $\mathfrak{A}$, will be called a form in the unknowns $y_{1}, \cdots, y_{n}$. Throughout our work we shall assume that the forms with which we deal belong to a fixed field $F$. Forms will be denoted by capital italic letters.

By the class of a form $F$, if $F$ involves one or more $y_{i j k}$ effectively, we mean the highest index $p$, such that some of the transforms $y_{p i}$ of $y_{p}$ or some of their derivatives $y_{p j k}$ are present in $F$. By forms of class zero, we mean forms free of the $y_{i j k}$, i.e., functions $f(x)$ of the field $\mathcal{F}$.

By the $\Delta$-order of $F$ in $y_{h}$, if $F$ involves some $y_{h j k}(j \geqq 0, k \geqq 0)$, we mean the order of the highest transform $y_{h q}$ of $y_{h}$ such that $y_{h q}$ or some of its derivatives $y_{h q k}$ are present in $F$.

By the $d$-order of $F$ in $y_{h l}$, if $F$ involves some $y_{h l k}(k \geqq 0)$, we mean the order of the highest derivative of $y_{h l}$ which is present in $F$.

From what precedes, it follows that we can associate four integers with each form in $y_{1}, \cdots, y_{n}$, not of class zero, viz.

(i) the class $p(p>0)$,

(ii) the $\Delta$-order $q$ in $y_{p}(q \geqq 0)$,

(iii) the $d$-order $r$ in $y_{p q}(r \geqq 0)$,

(iv) the degree $s$ in $y_{p q r}$ (in the ordinary algebraic sense; $s>0$ ).

These four numbers, in the succession described above,

$$
p, q, r, s,
$$

which will be called simply the quadruplet of the form, define the rank of the forms in the following sense.

If $A$ and $B$ are forms in the same unknowns $y_{1}, y_{2}, \cdots, y_{n}$, neither of class zero, if

and

$$
a_{1}, a_{2}, a_{3}, a_{4}
$$

$$
b_{1}, b_{2}, b_{3}, b_{4}
$$

are respectively the quadruplets of $A$ and $B$, then $A$ and $B$ are said to be of the same rank if $a_{i}=b_{i}$ for $i=1,2,3,4$. B will be said to be of lower rank than $A$ if, for the smallest index $i$ for which $a_{i}$ and $b_{i}$ are different, $b_{i}$ is less than $a_{i}$.

As for the forms of class zero, all of them are to be of the same rank and each of them of lower rank than a form of non-zero class.

Obviously, if $A$ is higher than $B$ and $B$ higher than $C$, then $A$ is higher than $C$. 
The following lemma can be proved very easily.*

Lemma. Every set of forms in $y_{1}, y_{2}, \cdots, y_{n}$ contains at least one form, the rank of which is not higher than that of any other form of the set.

\section{Ascending Sets}

Let $F$ be a form, not of class zero, with the quadruplet $p, q, r, s(p>0)$. A form $H$ is said to be reduced with respect to the form $F$ if for every $k \geqq q$ either

(a) $H$ does not involve $y_{p k}$, or

(b) the $d$-order of $H$ in $y_{p k}$ is less than $r$, or s.t

(c) the $d$-order of $H$ in $y_{p k}$ equals $r$ but the degree of $H$ in $y_{p k r}$ is less than

In the following we shall sometimes say " $H$ is of lower rank in $y_{p k}$ than $r, s$ " if $B$ has any one of the properties (a), (b), or (c) with respect to $y_{p k}$.

A set of forms

$$
A_{1}, A_{2}, \cdots, A_{m}
$$

is called an ascending set if either

(a) it consists of only one form $A_{1}$, not identically zero, or

(b) it contains more than one form, $A_{1}$ is of class greater than zero, and for $1 \leqq i<j \leqq m, A_{j}$ is reduced with respect to and of higher rank than $A_{i}$.

Let

$$
\begin{gathered}
A_{1}, A_{2}, \cdots, A_{k}, \\
B_{1}, B_{2}, \cdots, B_{l}
\end{gathered}
$$

be two ascending sets. $(A)$ will be called of higher rank than $(B)$ if either

(a) there exists a $j$, exceeding neither $k$ nor $l$, such that $A_{i}$ and $B_{i}$ are of the same rank for $i=1,2, \cdots, j-1$, but $A_{j}$ is higher than $B_{j}, \ddagger$ or

(b) $l$ is greater than $k$ and $A_{i}$ and $B_{i}$ have the same rank for $i=1,2, \cdots, k$. If $l=k$ and $A_{i}$ and $B_{i}$ have the same rank for $i=1,2, \cdots, k$, the ascending sets $(A)$ and $(B)$ are to be of the same rank.

Evidently, if $(A),(B),(C)$ are ascending sets, $(A)$ higher than $(B)$, and $(B)$ higher than $(C)$, then $(A)$ is also higher than $(C)$.

We shall now prove the following

* Cf. Ritt, loc. cit., p. 3, and Ritt-Doob, loc. cit., p. 506.

$\dagger$ It may be that for different $k$ 's we have different cases.

$\ddagger$ In the case $j=1$, that means simply that $A_{1}$ is higher than $B_{1}$. 
Lemma. If $\Phi$ is a system of ascending sets there exists in $\Phi$ at least one ascending set of lowest rank.

Consider the first forms of all ascending sets of $\Phi$. According to the lemma of $\S 2$, there are among them forms of lowest rank. Let $\Phi_{1}$ consist of all such ascending sets of $\Phi$ that begin with a form of that lowest rank. If all ascending sets of $\Phi_{1}$ have only one form, then each ascending set of $\Phi_{1}$ constitutes an ascending set of lowest rank in $\Phi$. $^{*}$ If not, consider the ascending sets of $\Phi_{1}$ which have at least two forms and choose those from among them whose second form is of lowest rank. Let the subsystem of $\Phi_{1}$ obtained in this way be called $\Phi_{2}$. If all ascending sets of $\Phi_{2}$ have exactly two forms each of them constitutes an ascending set of lowest rank in $\Phi$. If not, we continue in this fashion. If we can show that, after a finite number of steps, we reach a system $\Phi_{m}$ all ascending sets of which have exactly $m$ forms, then each ascending set of $\Phi_{m}$ constitutes an ascending set of lowest rank in $\Phi$ and the lemma will be proved.

In order to show that we must reach such a system $\Phi_{m}$, we consider any system $\Phi_{i}$ which we meet in the above process. We notice that all $i$ th forms of the ascending sets of $\Phi_{i}$ are of the same rank. Let $A_{i}$ be one of these forms and $p_{i}, q_{i}, r_{i}, s_{i}$ be the quadruplet of $A_{i}$. $\dagger$ Consider now any two consecutive systems $\Phi_{j}$ and $\Phi_{j+1}$. Since $A_{j+1}$ is of higher rank than and reduced with respect to $A_{j}$ we have only the following possibilities:

$$
\begin{aligned}
& p_{j+1}>p_{j}, \\
& p_{i+1}=p_{j} ; q_{j+1}>q_{j} .
\end{aligned}
$$

In case (b), since $A_{j+1}$ is of lower rank in $y_{p_{j} q_{j+1}}$ than $r_{j}, s_{j}$, either

$$
r_{j+1}<r_{j}
$$

or

$$
r_{j+1}=r_{j} ; s_{j+1}<s_{j} .
$$

The inequalities in $\left(b^{\prime}\right)$ and $\left(b^{\prime \prime}\right)$ now show that the case $(b)$ can occur only for a finite number of consecutive systems $\Phi_{i}$. Therefore, after a finite number of steps the case (a) must hold, i.e., the class $p_{i}$ must increase. But since $p_{i}$ can assume only the values $1,2, \cdots, n$ the process cannot be continued ad infinitum.

* This includes the case in which at least one set of $\Phi$ consists of a non-zero form of class zero. Every such ascending set is, of course, of lowest rank.

$\dagger$ We omit the trivial case, mentioned above, in which $A_{1}$ is of class zero; then $m=1$. 


\section{BASIC SETS}

If $\Sigma$ is a set of forms not all of which vanish identically, $\Sigma$ contains ascending sets (e.g., every form $F \not \equiv 0$ constitutes an ascending set). Among them, according to the lemma of $\$ 3$, there are ascending sets of lowest rank. Each such ascending set will be called a basic set of $\Sigma$.

If a form $F$ is reduced with respect to every form of an ascending set

$$
A_{1}, A_{2}, \cdots, A_{m}
$$

whose first form $A_{1}$ is not of class zero, then $F$ is said to be reduced with respect to the ascending set (1).

We prove the following lemma:

LeMma 1. If $\Sigma$ is a system of forms, (1) one of its basic sets, with $A_{1}$ not of class zero, and if $F$ is a non-zero form and reduced with respect to (1), then $\Sigma+F$ has a basic set lower than (1).

$F$ cannot be of the same rank as any of the forms in (1). If $F$ is lower than $A_{1}$ the ascending set $F$ is lower than (1). If not, let $j$ be the highest index such that $A_{j}$ is lower than $F(1 \leqq j \leqq m)$. Then $A_{1}, \cdots, A_{j}, F$ is lower than (1).

In exactly the same way one can prove

LEMмa 2. If $\Sigma$ is a system of forms, (1) one of its basic sets, with $A_{1}$ not of class zero, then $\Sigma$ contains no non-zero form which is reduced with respect to (1).

\section{REDUCTION OF FORMS}

If $A$ is any form in $y_{1}, y_{2}, \cdots, y_{n}$, we shall mean by the kth transform of the form $A$ the form which is obtained on replacing $x$ by $x+k$ in the coefficients of $A$ and in the unknowns, their transforms and their derivatives. The latter means that $y_{i j l}$ has to be replaced by $y_{i, j+k, l}$. The $k$ th transform will be denoted by $A^{(k)}\left[A^{(0)}=A\right]$.

Similarly, by the $m$ th derivative of a form $B$ we mean the $m$ th total derivative of $B$ with respect to $x$, the $y_{i j l}$ being regarded as functions of $x$. The $m$ th derivative of the $k$ th transform of $A$ will be denoted by $A^{(k, m)}\left[A^{(k, 0)}=A^{(k)}\right.$; $\left.A^{(0,0)}=A^{(0)}=A\right]$.

Evidently, $A^{(k, m)}$ can be interpreted also as the $k$ th transform of the $m$ th derivative of $A$. In any case, the first superscript of a form indicates the number of transformations, the second one the number of differentiations.

The properties (c) and (d) in the definition of a field ( $\$ 1)$ imply that the transforms and their derivatives of a form $A$ have coefficients in $\mathcal{F}$.

Let $A$ be a form, not of class zero, with the quadruplet $p, q, r, s$. Let $A$ be written as a polynomial in $y_{p q r}$ with coefficients which are forms in the $y_{i j k}$ lower than $y_{p q r}$. Then the derivative of this polynomial, i.e., $\partial A / \partial y_{p q r}$, 
will be called the separant of $A$, and its highest coefficient, i.e., the coefficient of the term $\left(y_{p q r}\right)$ in $A$, will be called the initial of $A$.

For the proof of the following reduction lemma we need a few facts about the quadruplet, the separant and the initial of the transforms and their derivatives of a form $A$, not of class zero. Let $p, q, r, s$ be the quadruplet of $A, S$ its separant, and $I$ its initial. Then the following table gives the facts that we will need in the proof. They will be seen to follow easily from the above definitions.

\begin{tabular}{lcc} 
Form & $A^{(k)}(k \geqq 0)$ & $A^{(k, m)}(k \geqq 0, m>0)$ \\
\hline Quadruplet & $p, q+k, r, s$ & $p, q+k, r+m, 1$ \\
Separant & $S^{(k)}$ & $S^{(k)}$ \\
Initial & $I^{(k)}$ & $S^{(k)}$
\end{tabular}

We finally notice that the separant and the initial of any form are of lower rank than the form itself.

We are now ready to prove the

Reduction LemMa. Let

$$
A_{1}, A_{2}, \cdots, A_{m}
$$

be an ascending set, with $A_{1}$ of class greater than zero, and let the separant and initial of the form $A_{i}$ be $S_{i}$ and $I_{i}$ respectively $(i=1, \cdots, m)$. If $G$ is an arbitrary form there exists a power product $T$ of the $S_{i}$, the $I_{i}$, and their transforms with non-negative integral exponents, such that by subtracting from $T \cdot G$ a suitable linear combination of the $A_{i}$, their transforms $A_{i}^{(k)}$ and the derivatives $A_{i}{ }^{(k, m)}$ of their transforms, with forms for coefficients, a form

$$
R=T \cdot G-\sum_{i, k, m} H_{i k m} A_{i}^{(k, m)}
$$

is obtained, which is reduced with respect to the ascending set (3).

It will suffice to consider the case in which $G$ is not reduced with respect to (3).

Let $j$ be the highest index such that $G$ is not reduced with respect to $A_{j}$. Let $p, q, r, s$ be the quadruplet of $A_{j}$. Since $G$ is not reduced with respect to $A_{j}$ there must exist at least one transform $y_{p h}$ of $y_{p}$, with $h \geqq q$, such that the rank of $G$ in $y_{p h}$ is not lower than $r, s$. We may assume that $y_{p h}$ is the highest such transform. Let $\rho$ be the $d$-order of $G$ in $y_{p h}$ and let $\sigma$ be the degree of $G$ in $y_{p h \rho}$. Then either 


$$
\rho=r ; \sigma \geqq s .
$$

We treat first the case (a).

We take the form $A_{j}^{(h-q, p-r)}$ where, according to our assumptions, $h-q \geqq 0, \rho-r>0$. The quadruplet of $A_{j}{ }^{(h-q, \rho-r)}$ is $p, h, \rho, 1$, its initial $S_{j}{ }^{(h-q)}$ (cf. table (2)). Dividing $G$ by $A_{j}{ }^{(h-q, \rho-r)}$ we get, with a suitable integer $v \geqq 0$,

$$
\left[S_{i}^{(h-q)}\right]^{v} \cdot G=C \cdot A_{j}^{(h-q, \rho-r)}+D .
$$

For the sake of uniqueness we take $v$ as small as possible.

$D$ is either identically zero or of lower degree in $y_{p h_{\rho}}$ than $A_{j}{ }^{(h-q, \rho-r)}$, i.e., $D$ does not contain $y_{p h p}$. Since higher derivatives of $y_{p h}$ than the $\rho$ th do not appear in $G$ and $A_{j}{ }^{(h-q, \rho-r)}, D$ cannot contain such derivatives either, so that the $d$-order of $D$ in $y_{p h}$ is less than $\rho$.

Furthermore, if $y_{k l}$ is any transform higher than $y_{p h}, D$ is not of higher rank in $y_{k l}$ than $G$. Suppose that were not true for some $y_{k l}$. Notice that $A_{j}{ }^{(h-q, p-r)}$ and $S_{j}{ }^{(h-q)}$ are free of $y_{k l}$. From (5) we conclude that $C$ would have to contain $y_{k l}$ in the same rank, say $\mu, \nu$, as $D$. Hence $C \cdot A_{j}{ }^{(h-q, \rho-r)}$ would contain a term involving the product $\left(y_{k l \mu}\right)^{n} \cdot y_{p h \rho}$, which could be balanced neither by $D$ nor by the first member of (5). This proves our statement.

We conclude first that $D$ is of lower rank than $r, s$ in each higher transform of $y_{p}$ than the $h$ th one, and secondly, that (in case $j<m$ ) $D$ is reduced with respect to $A_{j+1}, A_{j+2}, \cdots, A_{m}$.

If now $D$ involves $y_{p h}$ and the $d$-order of $D$ in $y_{p h}$, which was less than $\rho$, is still greater than $r$, i.e., if the case (a) still holds, we treat $D$ in the same way as we $\operatorname{did} G$, and, after a finite number (at most $\rho-r$ ) of such divisions, we reach a form $D_{1}$, which is either free of $y_{p h}$ (and its derivatives) or else of $d$-order less than or at most equal to $r$ in $y_{p h}$ but is, as $D$ was, of lower rank than $r, s$ in $y_{p, h+1}, y_{p, h+2}, \cdots$, and reduced with respect to $A_{j+1}$, $A_{j+2}, \cdots, A_{m}$.

If $D_{1}$ involves $y_{p h}$ and is of $d$-order exactly $r$ in $y_{p h}$ and of degree $\sigma \geqq s$ in $y_{p h r}$ (case (b)), then we take $A_{j}{ }^{(h-q)}$ which has, according to table (2), the quadruplet $p, h, r, s$ and the initial $I_{j}{ }^{(h-q)}$. By a division we get, with some integer $w \geqq 0$ which we shall take as small as possible,

$$
\left[I_{j}^{(h-q)}\right]^{w} \cdot D_{1}=B \cdot A_{j}^{(h-q)}+D_{2},
$$

where $D_{2}$ is either free of $y_{p h r}$ or of degree less than $s$ in $y_{p h r}$. 
Similarly, as we concluded for $D$ from (5), we now conclude for $D_{2}$ from (6) that $D_{2}$ does not contain any higher derivative of $y_{p h}$ than the $r$ th one, so that $D_{2}$ is of lower rank in $y_{p h}$ than $r, s$ and, furthermore, that $D_{2}$ is of lower rank than $D_{1}$ in each $y_{k l}$ which is higher than $y_{p h}$. This latter fact implies, as before, that $D_{2}$ is of lower rank than $r, s$ also in $y_{p, h+1}, y_{p, h+2}, \cdots$, and that it is reduced with respect to $A_{j+1}, A_{j+2}, \cdots, A_{m}$.

Using all the divisions of the type (5) and (6) made so far, we have a relation between $G$ and $D_{2}$ of the following type:

$$
\left[S_{i}^{(h-q)}\right]^{u} \cdot\left[I_{j}^{(h-q)}\right]^{w} \cdot G-\sum_{t=0}^{\rho-r} E_{t} \cdot A_{j}^{(h-q, t)}=D_{2}
$$

with non-negative integral $u$ and $w$.

If now $D_{2}$ is not yet reduced with respect to $A_{j}$ we must have, from what has been said about $D_{2}$, that there exists at least one transform $y_{p h^{\prime}}$ of $y_{p}$, with $q \leqq h^{\prime}<h$, such that $D_{2}$ is not of lower rank in $y_{p h^{\prime}}$ than $r, s$. We then apply the same method as above to the highest such transform $y_{p h^{\prime}}$ and reduce $D_{2}$ with respect to that transform. If necessary, we continue in this fashion until, after a finite number (at most $h-q+1$ ) of such steps, we have reached a form $D_{3}$, which is of lower rank than $r, s$ in $y_{p q}, y_{p, q+1}, \cdots$, hence reduced with respect to $A_{j}$, but also, as $D_{2}$ before, with respect to $A_{i+1}, \cdots, A_{m}$.

As relation between $G$ and $D_{3}$, we have

$$
\prod_{\lambda=0}^{h-q}\left[S_{i}^{(\lambda)}\right]^{u_{\lambda}}\left[I_{i}^{(\lambda)}\right]^{w_{\lambda}} \cdot G-\sum_{k=0}^{h-q} \sum_{m} F_{k m} A_{i}^{(k, m)}=D_{3},
$$

with non-negative integral exponents $u_{\lambda}$ and $w_{\lambda}$.

If $D_{3}$ is not yet reduced with respect to (3) let $i$ be the highest index such that $D_{3}$ is not reduced with respect to $A_{i}$. Then $i$ must be less than $j$. We then apply the above method in order to reduce $D_{3}$ with respect to $A_{i}$ and get a form $D_{4}$, reduced with respect to $A_{i}$ and also to $A_{i+1}, \cdots, A_{m}$. After a finite number (at most $j$ ) of such steps we finally reach a form $R$ which is reduced to the ascending set (3) and related to $G$ by the equation (4). We have thus proved the reduction lemma.

The form $R$ will be called in the sequel the remainder of the form $G$ with respect to the ascending set (3).

\section{Solutions}

We shall define what will be meant by a solution of a system of algebraic 
mixed difference equations. Our definition will be broad enough so as to admit also multiple-valued functions as solutions. ${ }^{*}$

Let $\mathfrak{A}$ be an open region of the type mentioned in $\$ 1$. Let $\Sigma$ be a system of forms in the unknowns $y_{1}, y_{2}, \cdots, y_{n}$, with coefficients meromorphic in $\mathscr{A}$ and belonging to the field $\mathcal{F}$ (cf. $\$ 2$ ).

Let $\phi(t)$ be a continuous complex function, defined for real $t \geqq 0$, such that all values $\phi(t)$ lie in $\mathscr{A}$ and $\phi(t)$ satisfies the equation

$$
\phi(t+1)-\phi(t)=1 .
$$

The function $x=\phi(t)$ defines a continuous path $\mathcal{L}$, lying entirely in $\mathfrak{A}$ and containing $x+1$ if $x$ lies on it. By a function analytic on $\mathcal{L}$, we shall understand a function $f(x)$ which is analytic at the point $x=\phi(0)$ and which can be continued analytically along $\mathcal{L}$, as one moves on it with increasing $t$. For the points $x$ on $\mathcal{L}, f(x)=f(\phi(t))=\psi(t)$ becomes thus a unique function of $t$. Using (7), we can and shall define $f(x+1)$ by

$$
f(x+1)=\psi(t+1) .
$$

It is obvious that $f(x+1)$ is also analytic on $\mathcal{L}$, and the same holds for all transforms and their derivatives of $f(x)$.

By a solution of the system $\Sigma$, we mean the entity composed of a path $\mathcal{L}$, as described above, and $n$ functions $y_{1}(x), y_{2}(x), \cdots, y_{n}(x)$ analytic on $\mathcal{L}$, such that the forms of $\Sigma$ vanish identically on $\mathcal{L}$ if the unknowns $y_{i}$ in them are replaced by the functions $y_{i}(x)$.

The totality of solutions of a system $\Sigma$ will be called the manifold of $\Sigma$.

If $\Sigma_{1}$ and $\Sigma_{2}$ are systems of forms such that every solution of $\Sigma_{1}$ is a solution of $\Sigma_{2}$, we shall say " $\Sigma_{2}$ holds $\Sigma_{1}$." $\dagger$ If two systems hold one another, i.e., if their manifolds are identical, they will be said to be equivalent.

We notice the following easy consequence of our definitions:

If $f(x)$ is analytic on $\mathcal{L}$, then $f(x+1) \equiv 0$ on $\mathcal{L}$ if and only if $f(x) \equiv 0$ on $\mathcal{L}$. Therefore, if $F$ is any form, $F$ has the same manifold as each of its transforms $F^{(k)}(k=1,2, \cdots)$.

\section{Completeness}

In this section we shall prove the following

THEOREM. Every infinite system of forms is equivalent to one of its finite subsystems.

We call a system which is equivalent to one of its finite subsystems a complete system. Systems which are not complete will be called incomplete.

* Cf. Ritt-Doob, loc. cit., p. 506, footnote.

$\dagger$ A system devoid of solutions is considered as being held by every system. 
We have to show that the assumption of the existence of incomplete systems leads to a contradiction.

We first prove the following

Lemma. Let $\Sigma$ be an incomplete system. Let

$$
F_{1}, F_{2}, \cdots, F_{m}
$$

be forms such that, by multiplying each form in $\Sigma$ by some power product* of the $F_{i}$ and their transforms $F_{i}{ }^{(k)}$, a system $\Lambda$ is obtained which is complete. Then at least one of the systems $\Sigma+F_{i}(i=1,2, \cdots, m)$ is incomplete.

Suppose each system $\Sigma+F_{i}$ is complete. Let $\Phi_{i}$ be a finite subsystem of $\Sigma+F_{i}$ which is held by $\Sigma+F_{i}(i=1,2, \cdots, m)$. Since $\Phi_{i}$ can be replaced by any finite subsystem of $\Sigma+F_{i}$ containing $\Phi_{i}$ in itself, we may assume that $\Phi_{i}$ is of the type

$$
G_{1}, G_{2}, \cdots, G_{l}, F_{i}
$$

(for $i=1,2, \cdots, m$ ), with the set

$$
G_{1}, G_{2}, \cdots, G_{l}
$$

independent of $i$ and the forms of (9) in $\Sigma$. We may, similarly, assume that the forms in $\Lambda$, obtained from the forms in (9) by the above described multiplication, form a system which is equivalent to $\Lambda$.

Since $\Sigma$ is incomplete there is a form $L$ in $\Sigma$ which does not hold (9). But the product of $L$ and some power product of the $F_{i}$ and their transforms holds (9). Since $F_{i}$ has the same manifold as its transforms (\$6) we conclude that $F_{1} \cdots F_{m} L$ holds (9). Since $L$ does not hold (9) there are solutions of (9) for which $L$ does not vanish, so that at least one of the $F_{i}$ must vanish. Hence, for at least one $i, L$ does not hold (8). This contradiction proves our lemma.

We shall now prove the completeness of all systems.

Suppose there are incomplete systems. Consider their totality and choose for each of them a basic set (\$4). According to the lemma of $\S 3$, there are incomplete systems whose basic sets are not higher than those of any incomplete system. Let $\Sigma$ be one of these systems, let

$$
A_{1}, A_{2}, \cdots, A_{m}
$$

be a basic set of $\Sigma$, and let $S_{i}$ and $I_{i}$, respectively, be the separant and the initial of $A_{i}(i=1,2, \cdots, m)$.

Then $A_{1}$ is of class greater than zero, for otherwise $\Sigma$ would have no solutions and would be equivalent to $A_{1}$. Hence, it would be complete.

* The power product may be different for different forms in $\Sigma$. 
We can then apply the reduction lemma (\$5) to each form $G$ of $\Sigma$ not in (10), and form the remainder $R$ of $G$ with respect to the ascending set (10). Let $T$ be the power product of the $S_{i}$, the $I_{i}$ and their transforms, used in the reduction of $G$ (cf. (4)). Let $\Omega$ be the system composed of the forms in (10) and the remainders $R$ of all forms $G$ of $\Sigma$ not in (10). Let $\Lambda$ be the system composed of the forms in (10) and the products $T \cdot G$ for all forms $G$ of $\Sigma$ not in (10).

Now the system $\Omega$ is complete. If not, $\Omega$ would contain non-zero forms, different from the forms in (10). Since such forms would be reduced with respect to (10), (10) could not be a basic set of $\Omega$, according to Lemma 2 of $\S 4$. The basic sets of $\Omega$ then would have to be lower than (10), so that $\Omega$ would be an incomplete system with a basic set lower than (10). Thus $\Omega$ is complete.

Since every form in $\Lambda$ which is not in (10) differs from the corresponding form in $\Omega$ by a linear combination of the $A_{i}$, their transforms and their derivatives, $\Lambda$ and $\Omega$ are equivalent and $\Lambda$ is also complete.

Applying the lemma of this section, we see that at least one of the $2 m$ systems $\Sigma+S_{i}$ and $\Sigma+I_{i}$ is incomplete. But every $S_{i}$ and $I_{i}$ is not identically zero and reduced with respect to (10). Consequently, according to Lemma 1 of $\S 4$, each of the systems $\Sigma+S_{i}$ and $\Sigma+I_{i}$ has a lower basic set than (10). This contradiction proves that incomplete systems cannot exist.

\section{DECOMPOSITION THEOREM}

A system $\Sigma$ of forms is said to be reducible if there exist two forms $G$ and $H$, such that neither $G$ nor $H$ holds $\Sigma$ but $G \cdot H$ does. Otherwise $\Sigma$ is called irreducible.

A system $\Sigma$ will be said to be equivalent to the set of systems $\Sigma_{1}, \Sigma_{2}, \cdots, \Sigma_{r}$ if every solution of $\Sigma$ is a solution of at least one of the systems $\Sigma_{i}$ and if every $\Sigma_{i}$ is held by $\Sigma$. In other words, $\Sigma$ is equivalent to the set $\Sigma_{1}, \Sigma_{2}, \cdots, \Sigma_{r}$ if the manifold of $\Sigma$ is the sum of the manifolds of the $\Sigma_{i}(i=1,2, \cdots, r)$.

We are now ready to prove the

Decomposition Theorem. Every system $\Sigma$ of forms in $y_{1}, y_{2}, \cdots, y_{n}$ is equivalent to a finite set of irreducible systems

$$
\Sigma_{1}, \Sigma_{2}, \cdots, \Sigma_{r}
$$

When those among the systems in (11) which are held by some other are suppressed, the set of the remaining systems, say

$$
\Sigma_{1}, \Sigma_{2}, \cdots, \Sigma_{s},
$$

will still be equivalent to $\Sigma$ but such that no system in (12) is held by any 
other. Such a decomposition of $\Sigma$ will be called a decomposition into essential irreducible systems.

The decomposition into essential irreducible systems is unique in the following sense: if (12) and

$$
\Omega_{1}, \Omega_{2}, \cdots, \Omega_{t}
$$

are two decompositions of $\Sigma$ into essential irreducible systems, then $t=s$ and, after a suitable permutation of the indices in (13), $\Omega_{i}$ and $\Sigma_{i}$ are equivalent for $i=1,2, \cdots, s$.

We prove first that every system can be decomposed into a finite set of irreducible systems. Suppose that were not the case for some system $\Sigma$. Then $\Sigma$ must be reducible. Let $G_{1}, H_{1}$ be such that $G_{1} \cdot H_{1}$ holds $\Sigma$ but neither $G_{1}$ nor $H_{1}$ does. Then $\Sigma$ is equivalent to the set of systems $\Sigma+G_{1}, \Sigma+H_{1}$.

At least one of these two systems cannot be equivalent to a finite set of irreducible systems. Suppose $\Sigma+G_{1}$ is not. Then, similarly, we find a form $G_{2}$ such that

(a) $G_{2}$ does not hold $\Sigma+G_{1}$,

(b) $\Sigma+G_{1}+G_{2}$ is not equivalent to a finite set of irreducible systems.

Continuing, we find an infinite sequence of forms

$$
G_{1}, G_{2}, \cdots, G_{k}, \cdots
$$

such that, for $i=1,2, \cdots, G_{i}$ does not hold the system $\Sigma+G_{1}+G_{2}+\cdots$ $+G_{i-1}$.

Let $\Lambda$ be the system composed of the forms in $\Sigma$ and all the forms in (14). According to the theorem of $\$ 7, \Lambda$ is equivalent to some finite subsystem $\Phi$ of $\Lambda$. Let $j$ be such that no $G_{i}$ in (14) with a higher index than $j$ belongs to $\Phi$. Then $\Lambda$ is equivalent also to its subsystem,

$$
\Sigma+G_{1}+G_{2}+\cdots+G_{j} .
$$

But that is impossible, since $G_{j+1}$ does not hold (15). This contradiction shows the existence of a decomposition.

We have now to show the uniqueness of the decomposition.

Let us consider the two decompositions (12) and (13) of $\Sigma$ into essential irreducible systems. We shall show first that $\Sigma_{1}$ is held by some $\Omega_{i}$. Suppose none of the systems in (13) holds $\Sigma_{1}$. Let $B_{i}$ be a form in $\Omega_{i}$ such that $B_{i}$ does not hold $\Sigma_{1}(i=1,2, \cdots, t)$. Then the form $B_{1} \cdots B_{t}$ would hold each $\Omega_{i}$, hence $\Sigma$ and also $\Sigma_{1}$. This is impossible since $\Sigma_{1}$ is irreducible.

Let $\Omega_{1}$ hold $\Sigma_{1}$. Similarly, $\Omega_{1}$ must be held by some $\Sigma_{i}$. Since $\Sigma_{1}$ is held by no $\Sigma_{i}$ with $i>1, \Omega_{1}$ is held by $\Sigma_{1}$. Consequently $\Omega_{1}$ and $\Sigma_{1}$ are equivalent. Continuing in this way, we get the result that each $\Sigma_{i}$ in (12) is equivalent to 
some $\Omega_{j}$ in (13) and vice versa, so that $t$ and $s$ must be equal. Thus the decomposition is unique in the sense described above.

\section{EXAMPLES}

1. Let us consider the system $\Sigma$ consisting of the following two equations in one unknown $y$ :

$$
\begin{array}{r}
y(x+1) \cdot y^{\prime}(x)^{2}+\pi^{2} \cdot e^{2 \pi i x} \cdot y(x)=0, \\
y(x+2)-y(x)=0 .
\end{array}
$$

Let $\mathfrak{F}$ consist of all rational functions in $e^{2 \pi i x}$ with complex coefficients.

Multiplying (17) by $y(x+1)$, we see that $y(x+1) \cdot y(x)$ is of period unity; the same holds for the function $e^{2 \pi i x}$. Hence, if we multiply (16) by $y(x+1)$ the second term of its left member becomes a function of period unity. Therefore also the first term does, i.e., $y(x+1)^{2} \cdot y^{\prime}(x)^{2}$ is of period unity. Hence, according to (17),

$$
y(x)^{2} \cdot y^{\prime}(x+1)^{2}-y(x+1)^{2} \cdot y^{\prime}(x)^{2}=0 .
$$

Thus for every solution of $\Sigma$, we have either

$$
y(x) \cdot y^{\prime}(x+1)-y(x+1) \cdot y^{\prime}(x)=0,
$$

or

$$
y(x) \cdot y^{\prime}(x+1)+y(x+1) \cdot y^{\prime}(x)=0 .
$$

The only solution of $\Sigma$ satisfying both (18) and (19) is

$$
y(x) \equiv 0 \text {. }
$$

Omitting this solution, we get from (18) and (17)

$$
\frac{y(x+1)}{y(x)}=\text { const. }= \pm 1 \text {. }
$$

The sign + leads to no solution of (16); the sign - gives the solutions

$$
y(x)= \pm i \cdot e^{\pi i x}
$$

of the system (16), (17), (18).

From (19), on the other hand, we obtain $y(x+1) \cdot y(x)$ as constant and, (20) being omitted, we may write

$$
y(x+1) \cdot y(x)=\frac{1}{c^{2}} .
$$


Then (16), multiplied by $y(x)$, gives the solution

$$
y(x)=c_{1} \cdot e^{ \pm c e^{\pi i x}}
$$

and from (22) we finally get $c_{1}{ }^{2}=1 / c^{2}$, hence

$$
y(x)=\frac{1}{c} \cdot e^{ \pm c e^{\pi i x}}
$$

with constant $c \neq 0$, as the solutions of the system (16), (17), (19).

(20), (21) and (23) are thus obtained as the manifold of $\Sigma$.

Determining $y^{\prime}(x+1) \cdot y^{\prime}(x)$ for each solution, we can decompose $\Sigma$ into the set of the following three systems, none of which is held by any other.

$$
\begin{array}{ll}
\Sigma_{1}: & (16),(17) \text { and } y^{\prime}(x+1) \cdot y^{\prime}(x)=0 . \\
\Sigma_{2}: & (16),(17) \text { and } y^{\prime}(x+1) \cdot y^{\prime}(x)+\pi^{2} e^{2 \pi i x}=0 . \\
\Sigma_{3}: & (16),(17) \text { and } y^{\prime}(x+1) \cdot y^{\prime}(x)-\pi^{2} e^{2 \pi i x}=0 .
\end{array}
$$

The manifolds of $\Sigma_{1}, \Sigma_{2}, \Sigma_{3}$ are (20), (21), (23) respectively.

We shall show that these three systems are irreducible, so that (24) is a decomposition of $\Sigma$ into essential irreducible systems.

We notice that all functions in our field $\mathcal{F}$ are of period unity. Hence if a form $F$ in $y$, with coefficients in $F$, has the solution $y(x)$, then also $y(x+1)$ is a solution of $F$.

Now, the irreducibility of $\Sigma_{1}$ is obvious. As for $\Sigma_{2}$, if a form $F$ vanishes for one solution in (21), say $y(x)=+i e^{\pi i x}$, it vanishes also for $y(x+1)=-i e^{\pi i x}$, which is the other solution in (21). This shows the irreducibility of $\Sigma_{2}$.

Similarly, let $A$ and $B$ be forms such that $A \cdot B$ holds $\Sigma_{3}$. Then by putting

$$
y(x)=\frac{1}{c} e^{+c e^{\pi i x}}
$$

in $A \cdot B$, this product vanishes identically in $x$ and $c$ so that either $A$ or $B$ does. If now $A$ vanishes for all solutions

$$
y(x)=\frac{1}{c} e^{+c e^{\pi i x}}
$$

then it must also vanish for

$$
y(x+1)=\frac{1}{c} e^{-c e^{\pi i x}}
$$

which are the other solutions in (23). Thus $A$ holds $\Sigma_{3}$ and the irreducibility of $\Sigma_{3}$ is proved. 
2. The following example shows that a pure differential equation, irreducible within the domain of differential equations (see Introduction), can be reducible, considered as a mixed difference equation.

Let $\mathcal{F}$ be a field of constants, to be determined later, but containing $\pi^{2}$. The form

$$
A=y^{\prime 2}+\frac{\pi^{2}}{4} y^{2}
$$

is algebraically irreducible if $i \pi$ does not belong to $F$. It can be shown, however, that in this case $A$ is also irreducible as a differential equation.

Now the solutions of $A$ are

$$
y(x)=c_{1} \cdot e^{\pi i x / 2}
$$

and

$$
y(x)=c_{2} \cdot e^{-\pi i x / 2},
$$

with $c_{1}$ and $c_{2}$ arbitrary constants.

For the solutions (25) we have

$$
y(x+1)-i \cdot y(x)=0,
$$

and for the solutions (26),

$$
y(x+1)+i \cdot y(x)=0 .
$$

The only solution which (27) and (28) have in common is $y(x) \equiv 0$. Hence if $i$ belongs to $F$ the system $A$ is reducible, considered as a mixed difference equation.

In order to reach our purpose, we have to choose the field of numbers $F$ in such a way that it contains $i$ and $\pi^{2}$ but not $\pi i$. Such a field can be obtained, for instance, by adjoining $i$ and $\pi^{2}$ to the field of rational numbers.

COLUMBia UnIVERSITY,

New YORK, N.Y. 